Western University

Scholarship@Western

2014

\title{
Waveguide Evanescent Field Scattering Microscopy: Bacterial Biofilms and their Sterilization Response via UV Irradiation
}

\author{
Qamrun Nahar \\ Western University \\ Frederik Fleißner \\ Western University \\ Jeremiah Shuster \\ Western University \\ Michael Morawitz \\ Western University \\ Christopher Halfpap \\ Western University
}

See next page for additional authors

Follow this and additional works at: https://ir.lib.uwo.ca/physpharmpub

Part of the Medical Physiology Commons, and the Pharmacy and Pharmaceutical Sciences Commons

\section{Citation of this paper:}

Nahar, Qamrun; Fleißner, Frederik; Shuster, Jeremiah; Morawitz, Michael; Halfpap, Christopher; Stefan, Mihaela; Langbein, Uwe; Southam, Gordon; and Mittler, Silvia, "Waveguide Evanescent Field Scattering Microscopy: Bacterial Biofilms and their Sterilization Response via UV Irradiation" (2014). Physiology and Pharmacology Publications. 138.

https://ir.lib.uwo.ca/physpharmpub/138 


\section{Authors}

Qamrun Nahar, Frederik Fleißner, Jeremiah Shuster, Michael Morawitz, Christopher Halfpap, Mihaela Stefan, Uwe Langbein, Gordon Southam, and Silvia Mittler 
Journal of Biophotonics, 7 (7) (2014) 542-551

\title{
Waveguide Evanescent Field Scattering Microscopy: Bacterial Biofilms and their Sterilization Response via UV Irradiation
}

\author{
Qamrun Nahar, ${ }^{1,2}$ Frederik Fleißner ${ }^{2,3}$ Jeremiah Shuster, ${ }^{4}$ Michael \\ Morawitz, ${ }^{2,3}$ Christopher Halfpap, ${ }^{2,3}$ Mihaela Stefan, ${ }^{5}$ Uwe Langbein, ${ }^{3}$ \\ Gordon Southam, ${ }^{6}$ and Silvia Mittler ${ }^{2 *}$ \\ ${ }^{1}$ Department of Electrical and Computer Engineering, The University of Western Ontario (Western \\ University), London, Ontario, N6A 5B9, Canada \\ ${ }^{2}$ Department of Physics and Astronomy, The University of Western Ontario (Western University), London, \\ Ontario, N6A 3K7, Canada \\ ${ }^{3}$ RheinMain University, Engineering Sciences, Am Brückweg 26, 65428 Rüsselsheim, Germany \\ ${ }^{4}$ Department of Earth Sciences, The University of Western Ontario (Western University), London, Ontario, \\ N6A 5B7 Canada \\ ${ }^{5}$ Trojan Technologies, 320 Gore Road, London, Ontario, N5V 4T7, Canada \\ ${ }^{6}$ School of Earth Sciences, The University of Queensland, St. Lucia, Queensland, 4072, Australia
}

Key Words: waveguide, evanescent field, scattering, microscopy, biofilm, UV sterilization 


\begin{abstract}
Waveguide Evanescent Field Scattering (WEFS) microscopy is introduced as a new and simple tool for label-free, high contrast imaging of bacteria and bacteria sensors. Bacterial microcolonies and single bacteria were discriminated both by their bright field images and by their evanescent scattering intensity. By comparing bright field images with WEFS images, the proportion of planktonic: sessile (i.e., 'floating': attached) bacteria were measured. Bacteria were irradiated with UV light, which limited their biofilm forming capability. A quantitative decrease in attachment of individual, sessile bacteria and in attached, microcolony occupied areas was easily determined within the apparent biofilms with increasing UV dose. WEFS microscopy is an ideal tool for providing rapid quantitative data on biofilm formation.
\end{abstract}

\title{
Introduction
}


Bacteria are the most metabolically diverse group of organisms found in all natural environments including air, water and soil. Bacteria commonly occur with food sources and are also found within and on our bodies. However, concerns exist over contamination of food, water, and air by pathogenic bacteria [1] that can enter our bodies through ingestion, inhalation, cuts or lacerations [2]. Therefore, there is an increasing interest in bacterial contamination and the need for anti-bacterial surfaces not only for application in the food industry but also for medical and hygienic purposes [3]. Over two million hospitalacquired cases of infection are reported annually in the USA, which lead to approximately 100,000 deaths annually and added nearly $\$ 5$ billion to U.S. healthcare costs $[4,5]$. Contamination of medical devices (e.g., catheters and implants) has been attributed to $45 \%$ of these infections [6]. Bacterial contamination of any surface typically begins with the initial adhesion of only a few cells that can then develop into a more structurally cohesive biofilm in less than 24 hours when provided with suitable nutrient conditions sustaining metabolism and cell division [7]. Therefore, a better understanding of bacterial adhesion to surfaces is important for technical surface development and in biomedical applications. However, the precise measurement of bacterial adhesion to surfaces are difficult and time consuming because bacterial cells typically occur on the micrometer-scale and their adhesion forces are generally low, typically $0.1-100 \mathrm{nN}$ [8]. Recent studies on the detection of bacteria on surfaces have focused on imaging systems such as optical [9] and fluorescent microscopy [10] to image the bacteria themselves or luminescence measurement of the presence of cells by ATP (adenosine triphosphate) detection systems [11]. Surface Plasmon Resonance (SPR) sensors [12], Nucleic Acid Detection [13], Optical Waveguide Lightmode Spectroscopy [14], Optical Leaky Waveguide Sensors [15], and 
Evanescent Mode Fiber Optic Sensors [16] have also been applied in order to detect biochemical toxins as signatures of bacteria.

Evanescent wave techniques (e.g., surface plasmon resonance spectroscopy and microscopy) are popular biochemical sensors that can detect changes in the refractive index architecture near a surface due to the presence of a binding analyte. In indirect optical biosensors (e.g., fluorescent detectors) a labeled molecule is generally tethered to the analyte to allow detection by fluorescence, chemi-luminescence, colorimetry or densitometry [17]. In recent years, Total Internal Reflection Fluorescent (TIRF) microscopy has been demonstrated to be an effective method for studying cell-substrate interactions that occur at surfaces and interfaces. Using TIRF microscopy, the behavior of various types of bacteria near surfaces has been characterized [18-20]. Total Internal Reflection Microscopy (TIRM) utilizes the basic technology of TIRF without any fluorescence dyes present in the sample by creating an optical contrast due to scattering. Recent studies have also demonstrated the use of TIRM for imaging microbial adhesion. Using this technology, the calculation of cell size and, surprisingly, the cell-substrate distance using oil-immersion objectives was possible [18-22] despite the uncertainty on the presence of extracellular polymeric substance (EPS).

In this current paper, we present a simple, label-free, imaging technique called Waveguide Evanescent Field Scattering (WEFS) microscopy suitable for studying the adsorption of bacteria to a surface and subsequent imaging of bacterial growth in three dimensions, forming structurally cohesive microcolonies. The waveguide evanescent field scattering technique was developed by Thoma et al. $[23,24]$ for technical structures on surfaces using ion exchanged waveguides. Later, Waveguide Evanescent Field 
Fluorescence (WEFF) microscopy was developed [25-29] as a straightforward alternative to (TIRF) microscopy for imaging ultrathin films and cell-substrate interaction using fluorescence dyes located in the plasma membrane.

In this present study, both concepts were combined by capturing exclusively scattered photons from bacteria present in the evanescent field of a waveguide that were either directly attached or located very closely to the waveguide. The WEFS technique utilized the exponentially decaying evanescent field of a propagating waveguide mode as the sole illumination source with a probing evanescent field thickness between 70-100 nm depending on the implemented waveguide. Attached bacteria generated strong signals whereas bacteria close to the surface but unattached generated weak signals. WEFS microscopy allowed for imaging of attached cells without the use of toxic labels or additional treatment, allowing us to examine the interaction between viable bacteria and the waveguide surface.

Nitrobacter, as a genus, oxidizes nitrite to nitrate to fulfill its energy needs and fixes carbon dioxide for its carbon requirements [30-35]. We have chosen Nitrobacter sp. 263 for this present study because it is a common, gram-negative bacterium found in natural waters and soil, where it typically grows as a biofilm. In aqueous solutions, this rod-shape bacterium grows and divides via binary fission employing one plane of cell division; therefore, a 'cell' can occur as individual cells (i.e., a single, rod-shaped cell) or a double cell (i.e., two, single cells still attached resulting from a recent cell division). Bacteria, such as Nitrobacter sp. 263, require solid substrates and nutrients in order to grow and form microcolonies on surfaces. Bacterial attachment is the first step towards the formation of microcolonies and the eventual development of cohesive 3D-biofilms. As these cells form 
microcolonies, extracellular polymeric substances (EPS) are secreted by cells and serve as the scaffolding for holding cell-assembles together.

\section{Experimental Details:}

\section{Waveguides}

This study employed step-index waveguides with holographic coupling gratings fabricated via ion milling [36]. The refractive index of the waveguide layer was 1.845 and layer thicknesses varied from $500-750 \mathrm{~nm}$. The substrate was fused silica (FQVIS2, Hebo, Germany) or BF33 (Schott, Mainz, Germany) glass with $\mathrm{n}=1.46$ and $\mathrm{n}=1.47$ at $\lambda=543.5$ $\mathrm{nm}$ [37]. The coupling gratings had a periodicity of $600-650 \mathrm{~nm}$. The waveguides were cleaned by submersion in 70\% ethanol (Sigma Aldrich, Canada) with sonication (Branson 2510, Branson, USA) for 20 min. They were then blown dry with nitrogen gas. An important aspect of this technology was the reusable nature of the waveguides.

\section{Bacterial Culture}

Nitrobacter sp. 263 was cultured on R2A $\left(\right.$ Difco $^{\mathrm{TM}}$ ) plates at room temperature (approximately $23^{\circ} \mathrm{C}$ ) for two weeks. For each colonization experiment, bacteria from one R2A plate were removed and suspended in $1 \mathrm{~mL}$ of filter-sterilized $(0.45 \mu \mathrm{m}$ pore-size $)$

distilled deionized water to produce an aqueous bacterial suspension (with $10^{6}$ bacteria/mL). A separate stock solution of R2B (i.e., broth / liquid culture medium) was made by dissolving R2A in sterile, distilled, deionized water and filtering this solution to remove the agar constituent leaving the dissolved nutrients for bacterial growth. 


\section{Colonization Experiment 1: Bacterial-Waveguide Attachment}

Bacterial attachment to the waveguide surface was achieved by placing a $50 \mu \mathrm{L}$ aliquot of the bacterial suspension on top of the waveguide for one hour at $37^{\circ} \mathrm{C}$. After bacteria attached to the surface, the waveguide was rinsed with sterile, distilled water and placed in a sterile Petri dish containing $20 \mathrm{~mL}$ of R2B and incubated for 24 hours at $37^{\circ} \mathrm{C}$ to allow the attached bacteria to grow. The samples were not agitated. After 24 hours incubation, the waveguides were examined using bright field microscopy to determine whether microcolonies had formed. Note that all images were taken of live cells in growth medium. Microcolonies were then analyzed using WEFS microscopy (see below).

\section{Colonization Experiment 2: Sterilized Bacterial-Waveguide Attachment}

Sterilization experiments were performed since this new microscopy technology has application in monitoring bacterial sterilization processes, e.g., in water works. Briefly, separate bacteria suspensions of $10 \mathrm{ml}$ (with $10^{6}$ bacteria/mL) were placed in a sterile, open glass dish and exposed in a low pressure collimate beam apparatus (LPCB) to induce sterilization $[39,40]$ at doses of $2,4,8,14,20$ and $30 \mathrm{~mJ} / \mathrm{cm}^{2}$ by increasing time to produce different doses [40]. This mode of UV photon sterilization was chosen for its common use in industrial applications and its ability to disrupt and dimerize neighboring DNA bases (thymine dimerization) that hinders bacterial growth but not viability [38,41]. Each 'sterilized' bacterial suspension, produced via the different dose exposures, was used in an identical colonization experiments as described for the first experiment.

It is important to note that prior to the first and second colonization experiment, separate aliquots of all bacterial suspensions $(1 \mathrm{~mL})$ were stained using BacLight ${ }^{\mathrm{TM}}$ 
(Invitrogen) Live-Dead stain and examined using fluorescence microscopy to confirm that the cells were viable.

\section{The Waveguide Evanescent Field Scattering Microscopy Set-up}

The setup used for the Waveguide Evanescent Field Scattering Microscopy (WEFS) consisted of a conventional inverted microscope (Zeiss axiovert 25, Oberkochen, Germany) equipped with a HeNe laser with a wavelength of $543.8 \mathrm{~nm}$ (Research ElectroOptics, $0.5 \mathrm{~mW}$ ) which was used as a polarized light source in either s- or p-polarization leading to TE or TM modes, respectively. The laser beam was coupled into a propagating waveguide mode via a grating [36]. The waveguide was mounted on the sample stage of the inverted microscope. During imaging, the bacteria were maintained in R2B (described above). A simple o-ring was used as a cuvette. The scattered light from the bacteria on the waveguide surface was captured by a microscope objective [10x (Olympus-U plan FLN), 20x (Olympus LUC plan FLN), 40x (ZEISS LD Plan-NEOFLUAR) and 63x (ZEISS LD Plan-NEOFLUAR)]. No filters were used. A digital camera (Diagnostic Instruments Inc, USA) and imaging software (Image Pro Express, Media Cybernetics) were used for image capture over a range of exposure times and for image analysis. A schematic of the set up can be found in [24].

\section{Results and Discussion}

A bright field image of individual Nitrobacter sp. 263 cells and microcolonies on a waveguide in R2B is shown in Fig.1a. Bacteria are represented as dark grey structures on the bright grey background (e.g., the waveguide surface). Single bacteria, rod-shape 
bacteria and microcolonies are visible. The corresponding WEFS image is shown in Fig.1b. Single bacteria and colonies can be seen as the bright white structures on the black background in the image.

Both microscopy techniques were capable of depicting individual bacterial cells and colony structures. However, in the bright field image more individual cells were visible in comparison to the WEFS image. Different regions within colonies and in areas where single bacteria occurred were selected from the WEFS image to determine a quantitative difference between the scattered intensities due to attached colonies versus single bacterial cells. Fig.1b shows some integration squares as examples. The integrated and areanormalized intensities of these regions (calculated using Matlab), represented by gray level values of the selected areas, produced variable scattering intensities: the single cells were systematically less bright than the intensities achieved for the areas representing colonies. These intensities were attributed to the difference in the relative number of bacteria that act as scattering centers within colonies in comparison to single cells that 'are initiating' surface colonization and to the presence of EPS in the microcolonies.

WEFS imaging and data analysis was performed systematically with increasing exposure time until over-exposure occurred (Fig.2). More bacteria, occurring as individual cells, appeared in the images with increased exposure time (see Fig.2a from top left to low right). Although the colonies gained intensity, the distribution of intensity within colonies did not change; furthermore, colony shape remained consistent. Due to the evanescent form of the illuminating light, bacteria that appeared at the lowest exposure time were bacteria that adhered closest to the surface. Bacteria that appeared with increased illumination time were located further away from the waveguide surface but still within the 70-100 nm 
evanescent field depth. It is not possible from a static image to determine whether or not bacteria were weakly attached to the surface or mobile in solution just above the waveguide surface. However, in a time laps experiment Brownian motion would not allow cells to 'rest' at any position for more than a fraction of a second unless there was some interaction with the waveguide surface.

In TIRF and WEFF microscopy, randomly scattered photons can lead to epifluorescence contributions in evanescent fluorescence images [11]. Therefore, randomly scattered photons from the waveguide can contribute to a signal from objects not directly located at or very near to the waveguide surface, especially when long integration times were chosen. In this study, there were only very small location shifts of single bacterial cells in the WEFS images with increased exposure time. Some cells may have moved during the course of the experiment since bacteria were still viable. However, this was negligible and did not affect the intensity per area data since they were within the integration frame. Note that the speed of the movement, in a time laps experiment, may be a measure for distinguishing between weakly attached bacteria and planktonic bacteria that have not metabolically 'committed' to irreversible colonization of the surface.

Fig.2b shows the integrated intensities of single bacteria and the colonies with respect to integration time. For all integration times, the intensity values of the colonies were significantly higher than single bacterial cells. The increase in intensity was linear in time for both single bacterial cells and colonies indicating that no additional objects were contributing to the signal with increasing integration time. Therefore, the random scattering contribution, due to general waveguide scattering, was not substantial. If substantial random scattering were observed, a nonlinear increase in intensity would have resulted. 
In Fig. 1 and 3, the number of individual bacterial cells was different in the bright field images compared to the WEFS images (i.e., the number of grey dots and lines is higher in the bright field images in comparison to the number of bright dots in the WEFS images). Using images with $100 \mathrm{~ms}$ exposure time, approximately $150 \pm 50$ individual bacterial cells were counted within a defined unit area in a bright field image; however, $12 \pm 1$ individual bacterial cells were counted in corresponding areas using the WEFS image. For the highest exposure time of $500 \mathrm{~ms}$ the number of individual bacteria in the WEFS image increased to $19 \pm 5$. Interestingly, the WEFS images did not depict any rodshape structures but only round features. Obviously bacterial attachment was initiated at the cell poles (versus 'lying' along the length of the cell) producing a round scattering fingerprint. Some of the single bacterial cells observed in the bright field images never appeared on the WEFS images even under prolonged exposure time. This indicates that these bacteria did not adhere to the waveguide surface; rather, these planktonic cells remained in suspension within the liquid growth medium. However, all colonies were attached to the waveguide surface and demonstrated the importance of bacterial colonization by a single cell that continues to grow and divide, eventually forming a colony on a surface (i.e., commitment to forming a biofilm) [43].

A bright field microscopy image (Fig.3a) and a WEFS microscopy image (Fig.3b) were compared at the highest magnification to assess the integrated intensity profiles within two selected regions within the WEFS image. One region was selected along a colony (Fig.3b, left frame) and a second region was selected in an area with no colonies but containing single bacterial cells (Fig.3b, right frame). In both frames, the intensity was integrated along the y-axis (height) of the frame and plotted versus distance (i.e., the width 
of the frame). The intensity profiles for the colony and the single bacteria are depicted in Fig.3c and d, respectively. Note the different intensity scales in each profile (i.e., 8,00016,000 a.u. for the colony and $0-4,000$ a.u. for the single bacterial cells). The colony exhibited a high scattering intensity larger than 10,000 a.u. along the entire width of the frame. This observation is attributed to the presence of many scattering centers occurring in close proximity to one another. In addition extracellular polymeric substance was present and also contributed to the overall scattering intensity by filling the available volume between the cells in the evanescent field [39]. This observation also depicted a high local intensity fluctuation: different bacterial cells were located at different positions within the evanescent field causing variations in scattering strength. The density of the scattering objects was not constant in this region of the colony on the waveguide surface and indicated that bacterial attachment density within the colony, (i.e., distance above the waveguide surface), was heterogeneous within the first $100 \mathrm{~nm}$ from the waveguide surface. This behavior was observed in all colonies (Fig. 1b).

The intensity profile from the region containing individual bacterial cells (Fig.3b) was different in comparison to the region containing the colony. The overall intensity of this region was approximately one order of magnitude smaller and in some instances no WEFS intensity was detected. Fluctuations up to 500 a.u. (i.e., between 6 and $11 \mu \mathrm{m}$ distance) were interpreted as background noise, maybe because of the presence of some extracellular polymeric substance. Three individual bacterial cells produced separate scattering intensities represented by the distinctive peaks occurring at approximately 2.5 , 5 and $12 \mu \mathrm{m}$ distance (Fig.3b, and d). Both images (Fig.3a and b) confirm the presence of these three bacteria. It was not quite clear at this point why individual bacterial cells that 
have a size in the micrometer-scale, and are therefore one order of magnitude larger than the penetration depth of the evanescent field, produced different scattering intensities on a comparable scattering-spot size. The differences in peak height may be attributed to different bacteria sizes, orientation on the waveguide surface or to the presence of EPS. There are various possibilities: The bacterium producing the highest peak was a rod-shape bacterium attached with its long axis perpendicular to the waveguide surface, whereas the other two peaks have been produced by single bacterial cells. All three peaks were produced by single cells, however the highest peak arose from a single cell that attached with its longer axis parallel to the waveguide surface occupying a higher scattering volume whereas the other two were attached with one of their poles occupying less volume in the evanescent field. The precise location of the bacterial cell in the evanescent field might have dictated the scattering strength and therefore gave a hint on the attachment quality. However, it is unclear whether the bacteria have secreted EPS, which could contribute to the scattered signal. To find a solid interpretation for the intensity differences more comparative investigation are necessary and a thorough theoretical investigation including size, shape, orientation, distance from the surface and possible presence of EPS.

At the highest possible magnification, a bright field image and a WEFS image were compared. Various differences and comparison issues were observed:

1. In the bright field image a single bacterial cell and a very small colony with three or four cells, a beginning colony, were identified on the left and right side of a bright field image, respectively (Fig.4a).

2. In the corresponding WEFS image (Fig.4b) the colony was identified by the detection of a brightly scattering bacterial cell adhered to the surface that was associated 
with two, 'medium brightness' scattering bacteria located nearby; however, in a different geometry in comparison to the bright field image.

3. The focal plane in the bright field image and the WEFS image (Fig.4) were not identical. The bright field image depicts the uppermost three cells of the beginning colony whereas the WEFS image shows the lowest, closest to the waveguide positioned bacterial cells.

4. An individual bright spot in the middle of the WEFS image appeared where no cell was observed in the corresponding bright field image. This particular bacterium was weakly scattering and was therefore not strongly attached to the surface (Fig.4b and c).

5. The single bacterial cell observed on the left hand side on the bright field image was not observed in the corresponding WEFS image. It is important to note that no signal was detected for this cell at this location in the integrated intensity scan; therefore, it is interpreted as being unattached.

6. Samples were always focused on the lowest possible plane during bright field microscopy. However, the Zeiss axiovert 25CFI has fairly coarse focusing mechanics and a relative large depth of field; therefore, it was not exactly clear where the precise focal plane in a bright field image was located. Therefore the images taken were not necessarily in focus for bacterial cells directly attached to the waveguide surface but of bacteria that occurred above the WEFS focal plane, including bacteria within the 3D microcolonies.

The single bacterial cell (Fig.4a) was in better focus relative to the beginning colony and is therefore interpreted to be located at a further distance in excellent agreement with the WEFS image where the single bacterial cell is not observed (see 5.). 
7. The beginning colony clearly demonstrated three cells in the WEFS image however with cell locations completely different from the bright field image. The reason for this was attributed to the different focal plane of each respective image (see 3.). The three bacterial cells on the top of the colony (bright field) are arranged differently in comparison to the three lowest bacterial cells located close to the waveguide surface (WEFS). The colony produced a strong integrated scattering intensity peak with a shoulder on the left hand side in the intensity scan of the WEFS image (Fig.4c). The main peak was attributed to the scattering of two bacteria, one strong and one medium in intensity (Fig. $4 b$ ) while the shoulder was attributed to the bacterial cell located slightly to the left of the main scatterer. The initiation of colony formation required a single cell (i.e., a parent cell) to securely attach to the surface. The central cell with the highest scattering intensity must be that parent cell from which the colony started growing and the cell responsible for the committed and irreversible colonization of the surface. The two side-on bacterial cells exhibited less scattering intensity relative to the strongly attached cell. This means they were located in the evanescent field but not necessarily attached to the waveguide, however they are presumably attached to the parent cell at a position very close to the waveguide surface. They cannot be planktonic as they are within the colony.

We conclude the development here is a $3 \mathrm{D}$ process where rotational orientations occurring after the first two cell division steps of the 'parent (attached) bacterium' produced daughter cells that were not yet directly attached to the surface.

8. The single bacterium observed in the WEFS micrograph only (discussed above) is represented by the small intensity peak occurring at the $4 \mu \mathrm{m}$ mark that was roughly half the height of the shoulder. It was located close enough to the waveguide surface to produce 
a detectable scattering signal but was not adhered to the waveguide surface. The cells that comprised the colony had higher scattering intensities as represented by the high-intensity peak and the associated peak shoulder. Since it was not observed in the bright field image, it was not located in the focal plane depicting the top of the starting colony. Therefore, this single bacterial cell was likely suspended and could have represented the process of a bacterium beginning to adhere to the waveguide surface.

9. The wavy intensity structure between 0-3 $\mu \mathrm{m}$ distances was attributed to noise since the waveguide itself can produce minor scattering (surface irregularities and refractive index fluctuations) that can contribute to localized intensity fluctuations. Therefore, in order to avoid artifact detection in quantitative data analysis a threshold intensity value needs to be defined above which the scattering signal of the specimen is measured.

Studies on the attachment of bacteria onto surfaces using Waveguide Evanescent Field Scattering Microscopy might be a quick new method for investigations regarding bacterial sterilization treatment. We hypothesize that non-potent, sterilized cells do not attach to surfaces and do not form microcolonies. Therefore, we have treated identical bacteria sample batches with different UV doses $\left(2,4,8,14,20\right.$ and $\left.30 \mathrm{~mJ} / \mathrm{cm}^{2}\right)$. After the UV illumination the viability was measured. The UV illumination did not result in bacterial death. As a control, one sample was left without UV treatment. All bacteria illuminated with different UV doses and the control were cultured identically and examined using WEFS microscopy after 24 h. Fig. 5 shows a series of WEFS and bright field images of the control and UV treated bacteria. 
The relative signal attributed to attached colonies and individual bacteria on the waveguide surface decreased as exposure to UV illumination was increased (Fig.5). It is significant to note that the highest dose of $30 \mathrm{~mJ} / \mathrm{cm}^{2}$ was not sufficient to completely prevent bacterial attachment. Both WEFS and bright field microscopy demonstrated that the highest dose resulted in the attachment of primarily individual bacteria, demonstrating that while attachment still occurred with increasing UV-dose, microcolony formation was prevented.

In order to yield quantitative data, a Matlab program was written to investigate the intensity distribution of each WEFS image and to calculate the percentage of area (i.e., pixels with signals above the defined threshold) occupied by bacteria (i.e., individual cells and cells comprising distinct colonies). In addition, the percent area of colonies that appeared 'connected' and occupied an area with a particular number of pixels was calculated. These results indicated that a very large number of colonies occupying a small area were present and with increasing occupied area the number of colonies decreased, as expected. Fig. 6 shows the percentage of area on a sample occupied by bacteria versus the applied UV dose. Although the percentage of surface area with attached bacteria was decreasing exponentially, it did not reach zero. Bacteria were still attached to the waveguide surface despite the UV treatment. A rough extrapolation of the exponential curve suggests that a dose of $>100 \mathrm{~mJ} / \mathrm{cm}^{2}$ would be required to completely hinder all bacterial attachment to the surface. If this had been a water sterilization experiment, at least a dose of $200 \mathrm{~mJ} / \mathrm{cm}^{2}$ (double safety) should be applied before release to the user. A threshold area of 400 pixels was chosen (as a definition of a minimal size for distinct colonies) to compare the percent occupied area versus the available substrate surface on all 
images for all investigated doses for colonies. The area possessing these distinct colonies was about half of the area occupied by all bacteria and decayed exponentially with increasing dose. The decay rate of the distinct colonies was faster than for all bacteria. After a dose of $14 \mathrm{~mJ} / \mathrm{cm}^{2}$ was applied, none of these large, distinct colonies were found. When the area pixel threshold was defined smaller, more colonies 'appeared' and a higher dose for extinction was necessary, and vice versa (data not shown). This dose responsemicrocolony curve highlights the use/optimization potential of this new technique in developing controls on sterilization success.

\section{Conclusion}

This study demonstrated that WEFS microscopy is an effective tool for creating high contrast images analyzing the attachment of bacteria to surfaces and their subsequent development into microcolonies leading to biofilm formation. WEFS microscopy is capable of detecting direct bacterial attachment versus suspended cells or cells attached to a parent cell close to the surface via the intensity of the light they scatter when they were located in the evanescent field of the waveguide. Results from this present study suggest that the cell culturing applied here together with this WEFS technology has the capacity to detect bacterial attachment onto the waveguide after 1 hour, maybe even earlier.

The high concentration of bacteria in a colony and the presence of EPS provided large scattering intensities delivering a very high optical contrast relative to non-colonized areas. The WEFS contrast was enhanced in comparison to the contrast delivered by the bright field images. 
The comparison between WEFS images and bright field images demonstrated that a distinction between suspended and attached bacteria was possible with WEFS microscopy but not with bright field microscopy. The simple counting of bacteria numbers from both imaging modes delivers a measure of suspended : attached cells close to the surface. This is a very straightforward method with only statistical errors. No artifacts were found by changing the exposure time. WEFS images proved that even if there were numerous bacteria visible in the bright field images, the bacteria within the microcolonies were in close proximity to the surface or in direct contact with the substrate. In contrast, most of the individual bacterial cells were suspended (i.e., observed above the surface) demonstrating that cell division from the microcolonies into the fluid phase was an active process, producing single bacterial cells that could serve as new colonizers. (Note, the experiments were started $24 \mathrm{~h}$ after cell attachment had been confirmed, attached cells had been rinsed off and culturing was performed in medium without any additional cells.)

The rod-shape bacteria that were visible in the bright field images did not appear in the WEFS images: the bacteria attached by their poles in this non-agitated culture. The sterilization experiments proved our hypothesis that with less cell potency less cell attachment occurred. In addition, the colony formation probability decreased.

The area-normalized scattering intensity of colonies is significantly higher in comparison to areas containing individual attached bacteria. For a straight forward application of WEFS technology in bacterial detection in liquid or air environments or biofilm formation (desired or undesired) sensing, the degree of bacterial attachment on a surface can be determined by a simple intensity measurement integrating the scattered light along the entire waveguide surface and calculate how much of the inspected area is 
occupied by attached bacteria. In an on-line monitoring fashion the integrated scattering intensity of a waveguide chip can be watched and above a certain threshold, the sample can then be removed from its sampling environment and inspected with WEFS and other microscopies in detail to learn about a possible thread.

WEFS microscopy is a label free method and the waveguides are fairly robust with respect to chemical environment and temperature. It can therefore be applied under many circumstances for the detection of bacteria, their viability and their potency, e.g., the colonization of bacteria from natural or industrial sources of water (or wastewater). This technique can also contribute to long-term or time-lapse studies of cells because there is no photo-bleaching involved in imaging. Time-laps WEFS microscopy could give fundamental information of the colonization process at its earliest stage.

Most importantly, WEFS provides a novel method to study the quality and longterm stability of anti-bacterial coatings that can be easily applied to the waveguide surface.

\section{References}

[1] K. E. Sapsford, and L. C. Shiver-Lake, in M. Zourob, S. Elwary, and A.P.F. Turner, eds, Principles of Bacterial Detection: Biosensors, Recognition Receptors and Microsystems. (Springer, 2008) 109-123.

[2] J. Pizarro-Cerda, and P. Cossart. Cell 124, 715-725 (2006).

[3] J. D. Oliver. J. Microbiol. 43, 93-100 (2005).

[4] A. E. Madkour and G. N. Tew, Polym. Intl. 57, 6 (2008).

[5] A. E. Madkour, J. M. Dabkowski, K. Nusslein, and G. N. Tew, Langmuir 25, 10601067 (2009). 
[6] W.E. Stamm, Annals of International Medicine 89, 764-769 (1978).

[7] E.M. Hetrick, and M. H. Schoenfisch, Chem. Soc. Rev. 35, 780-789 (2006).

[8] G.E. Christianson, in Molecular Adhesion and Its Applications, The Sticky Universe, ed. Kevin Kendal, 275-303 (2004).

[9] K. Vasilev, J. Cook, and H. J. Griesser, Expert Rev. Med. Devices 6, 553-567 (2009).

[10] L. Pires, K. Sachsenheimer,T. Kleintschek, A. Waldbaur, T. Schwartz, B.E. Rapp, Biosensors and Biolectronics 47, 157-163 (2013).

[11] N.P. Pera, A. Kouki, S. Haataja, H.M. Branderhorst, R.M.J. Liskamp, G. M. Visser, J. Finne, and R. J. Pieters, Org. Biomol. Chem. 8, 2425-2429 (2010).

[12] A.D. Taylor, J. Ladd, J. Homola, and S. Jiang, in M. Zourob, S. Elwary, A. Turner, eds., Principles of Bacterial Detection: Biosensors, Recognition Receptors and Microsystems XXXII (Springer, 2008) 83-108.

[13] M. Schmidt, M.K. Hourfar, S.-B. Nicol, A. Wahl, J. Heck, C. Weis, T. Tonn, H.-P. Spengler, T. Montag, E. Seifried, W.K. Roth. Transfusion 46, 1367-1373 (2006).

[14] I.R. Cooper, S.T. Meikle, G. Standen, G.W. Hanlon, and M. Santin, J. Microbiol. Meth. 78, 40-44 (2009).

[15] M. Zourob, S. Mohr, B. J. T. Brown, P. F. Fielden, M. B. McDonnell, and N. J. Goddard, Biosensors and Bioelectronics 21, 293-302 (2005).

[16] A. Mazhorova, A. Markov, A. Ng, R. Chinnappan, O. Skorobogata, M. Zourob, and M. Skorobogatiy, Opt. Express 20, 5344-5355 (2012).

[17] M. Yang, K. E. Sapsford, N. Sergeev, S. Sun, and A. Rasooly, Proc. SPIE 7167,716702 (2009). 
[18] L.V. Smith, L. K. Tamm, and R. M. Ford, .Langmuir. 18, 5247-5255 (2002).

[19] M.A.S. Vigeant, M. Wagner, L. K. Tamm, and R. M. Ford, Langmuir 17,2235$2242(2001)$.

[20] H.M. Grandin, B. Städler, M. Textor, and J. Vörös, Biosensensors and Bioelectronics 21, 1476-1482 (2006).

[21] G.D. Bryne, M. C. Pitter, J. Zhang, F. H. Falcone, S. Stolnik, and M. G. Somekh, J. Microsc. 231, 168-179 (2008).

[22] T. Velinov, . Asenovska, D. Marinkova, L. Yotova, S. Stoitsova, M. Bivolarska, and L. Stavitskaya, Colloids and Surfaces B: Biointerfaces 88, 407-412 (2011).

[23] F. Thoma, U. Langbein, S. Mittler-Neher, Optics Communications 134, 16-20 (1997).

[24] F. Thoma, F., J. Armitage, H. Trembley, B. Menges, U. Langbein, and S. MittlerNeher, Proceedings of SPIE 3414, 242-249 (1998).

[25] A. Hassanzadeh, M. Nitsche, S. Armstrong, J. Dixon, U. Langbein, and S. Mittler, Applied Physics Letters 92, 233503 (2008).

[26] A. Hassanzadeh, A., H. Kan Ma, S. Armstrong, S. J. Dixon, S. M. Sims, S. Mittler, Proceedings of SPIE 7322, 73220 A1-A10 (2009).

[27] A. Hassanzadeh, A., M. Nitsche, S. Armstrong, N. Nabavi, R. Harrison, S. J. Dixon, U. Langbein, and S. Mittler, J. Biomed. Opt. 15, 036018-1 - 036018-7 (2010).

[28] A. Hassanzadeh, and S. Mittler, J. Biomed. Opt. 16, 04602 (2011).

[29] A. Hassanzadeh, S. Armstrong, S. J. Dixon, and S. Mittler, Applied Physics Letters 94, 033503 (2009). 
[30] S.R. Starkenburg, P.S. Cain, L.A. Sayaveda-Soto, L. Hauser, M.L. Land, F.W. Larimer, S.A. Malfatti, M.G. Klotz, P.J. Bottomley, D.J. Arp, and W.J. Hickey, Appl. Environ. Microbiol. 72, 2050-2063 (2006).

[31] E. Spieck, and E. Brock, in G.M. Garrity, D.J. Brenner, N.R. Krieg, and J.T. Staley (eds.) Manual of Systematic Bacteriology, Vol. 2, (Springer 2005) page 52, Springer.

[32] H.A. Painter, Water Research. Vol. 4, pp., (Pergamon Press 1970) 393-450.

[33] G. Zavarzin, and R. Legunkova, J. gen. Micobiol. 21, 186-190 (1959).

[34] J. Meikeljohn, Journal of Soil Science 4, 59-68 (1953).

[35] P. Stoodley, K. Sauer. D.G. Davies and J.W. Costerson, Annual Reviews in Microbiology 56, 187-209 (2002).

[36] X. Mei, R. Moshrefzadeh, U.J. Gibson, G.I. Stegeman and C. T. Seaton, Applied Optics 24, 3155-3161 (1985).

[37] C. Halfpap, M. Morawitz, A. Peter, N. Detrez, S. Mittler, and U. Langbein, DGaO Proceedings 02872012 (2012).

[38] M. Berney, F. Hammes, F. Bosshard, H.-U. Weilenmann, and T. Egli, Applied and Environmental Microbiology 73, 3283-3290 (2007).

[39] R.P. Hedrick, B. Petri, T.S. McDowell. K. Mukkatira, and L.J. Sealey, Diseases of Aquatic Organisms 74, 113-118 (2007).

[40] J. Kuo, M. Asce, C.-L. Chen, and M. Nellor Journal of Environmental Engineering 129 774-779 (2003).

[41] B. Durbeej, and L.A. Eriksson, Journal of Photochemistry and Photobiology A: Chemistry 152, 95-101 (2002). 
[42] J.R. Bolton, and K.G. Linden, Journal of Environmental Engineering-ASCE 129, 209-215 (2003).

[43] J.W. Costerton, Z. Lewandowski, D. E. Caldwell, D. R. Korber, and H. M. LappinScott, Annu. Rev. Microbiol. 49, 711-745 (1995).

Figure Captions:

Fig.1 (a) Bright field image (10x) and (b) WEFS image (10x) of Nitrobacter sp. 263 (exposure time $300 \mathrm{~ms}, \mathrm{TM}_{2}$ mode). The squares in the image depict areas where average intensities have been measured. Scale bars equal $200 \mu \mathrm{m}$.

Fig.2 WEFS images (10x) at exposure time (a) $150 \mathrm{~ms}$, (b) $200 \mathrm{~ms}$, (c) $250 \mathrm{~ms}$ and (d) $300 \mathrm{~ms}$. (e) Integrated intensity/area from colonies (black) and single cell (noncolony, white) areas versus exposure time. Scale bars equal $200 \mu \mathrm{m}$.

Fig.3 (a) Bright field image of bacteria taken with the $63 x$ objective and (b) the corresponding WEFS image. The small white boxes in the images highlight regions 
of identical area possessing a colony versus a non-colonized region with single bacteria. The intensity profiles (integrated along the y-axes) in the highlighted regions from the WEFS images were measured and plotted for (c) the colony and (d) the single bacteria area. Scale bars equal $10 \mu \mathrm{m}$.

Fig.4 (a) Bright field image of a single bacterium and a starting bacteria colony taken with the highest magnification (63x), (b) the corresponding WEFS image at $t=$ $2000 \mathrm{~ms}$ integration time (close to over exposure) and (c) the integrated intensity along the WEFS image. Scale bars equal $1 \mu \mathrm{m}$.

Fig.5 WEFS and bright field microscopy images (40x) of UV illuminated, sterilized bacteria after $24 \mathrm{~h}$ of culturing: a) and e) control: $0 \mathrm{~mJ} / \mathrm{cm}^{2}$ ), b) and f) $8 \mathrm{~mJ} / \mathrm{cm}^{2}$, c) and g) $20 \mathrm{~mJ} / \mathrm{cm}^{2}$, d) and h) $30 \mathrm{~mJ} / \mathrm{cm}^{2}$. The scale bar is $50 \mu \mathrm{m}$.

Fig.6 Percentage of occupied area of bacteria (single bacteria, starting colonies, and distinct colonies: $\square$ ) versus applied UV dose. From these, the percentage of colonies with a minimum area size of 400 pixels versus the UV dose $(\bullet) .100 \%$ are all pixels, the entire area, of the image.

\section{Figures}

FIG.1 


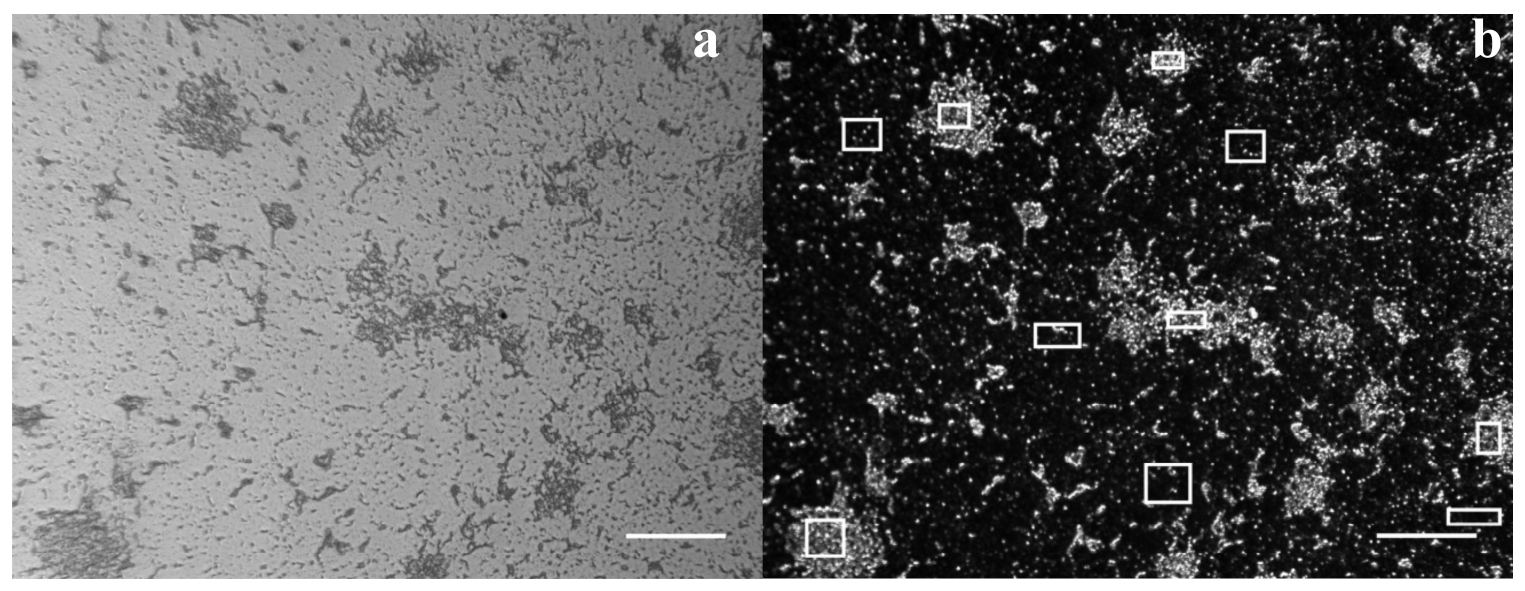

FIG.2
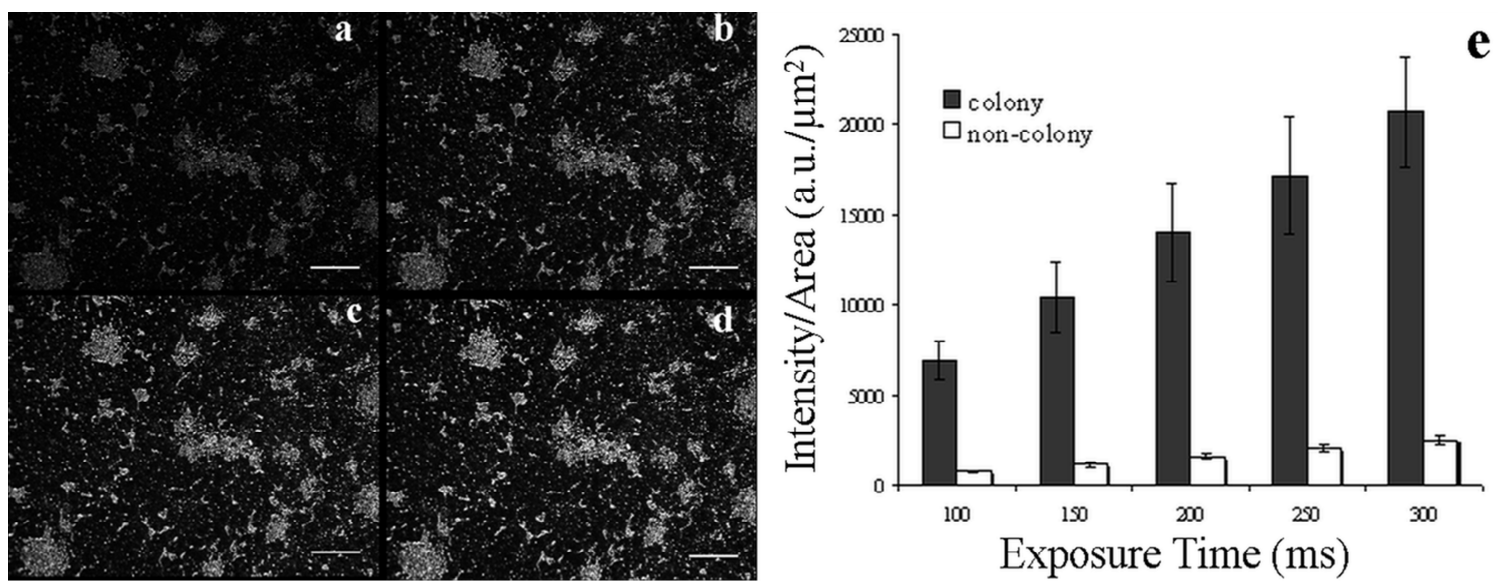

FIG.3 


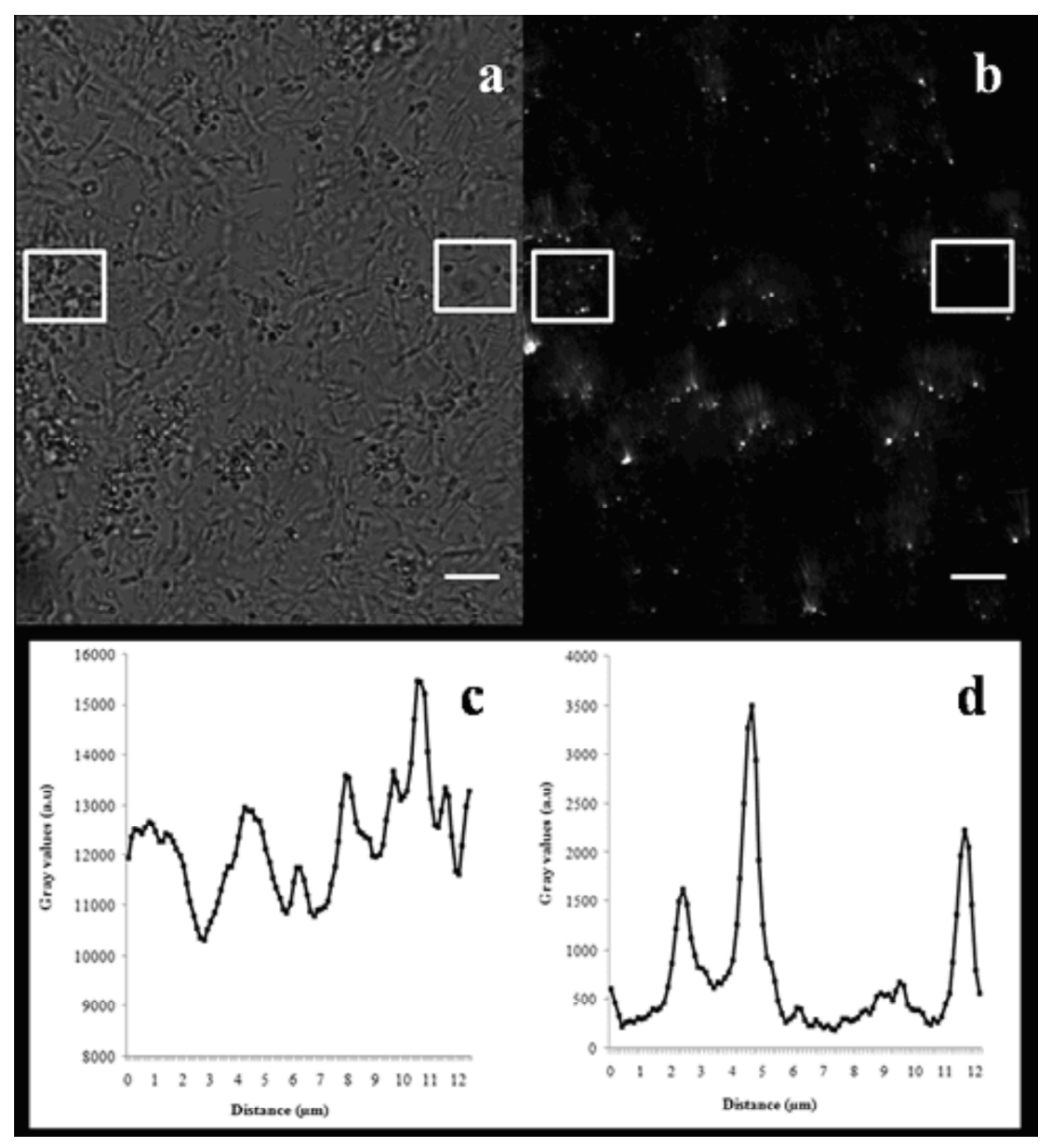

FIG.4 


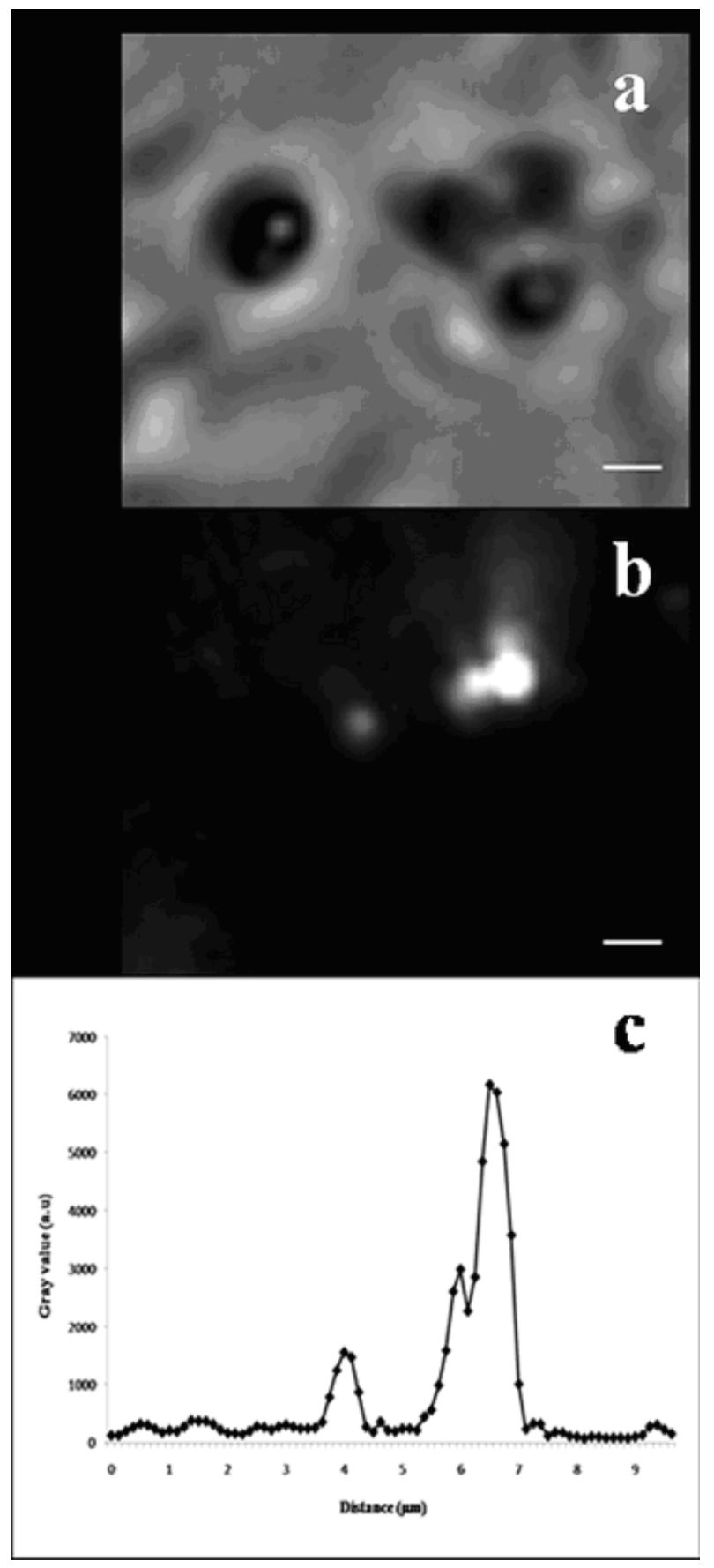

FIG.5 

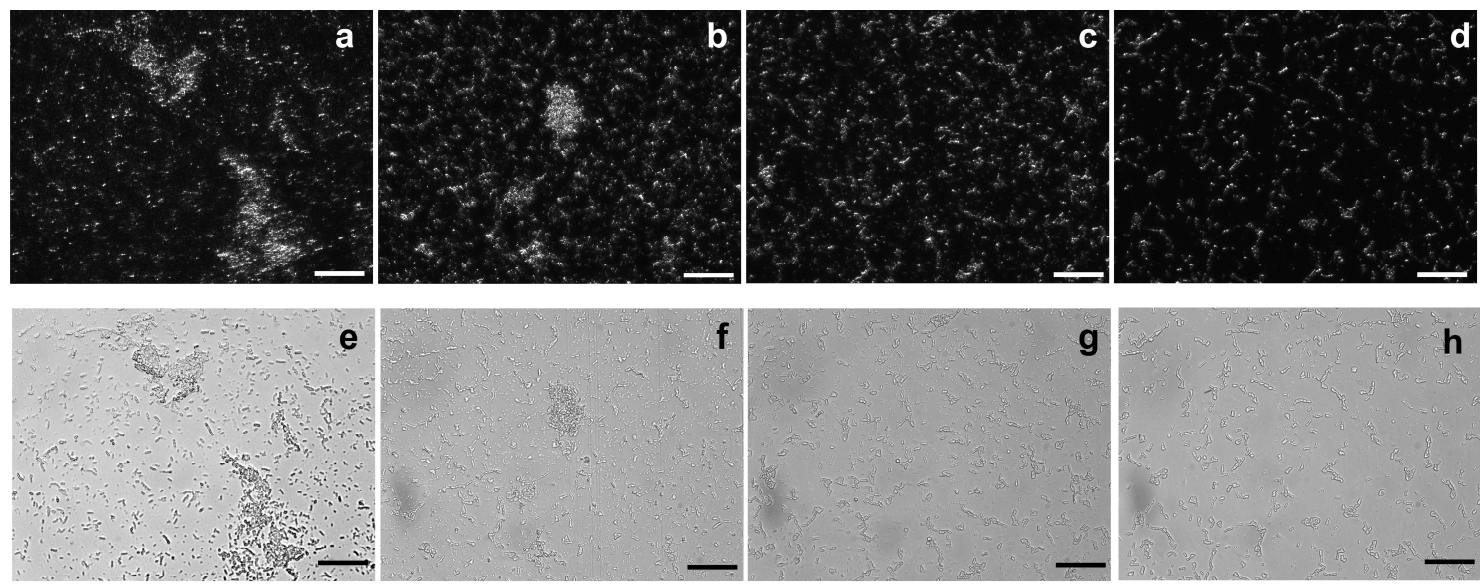

h

FIG.6

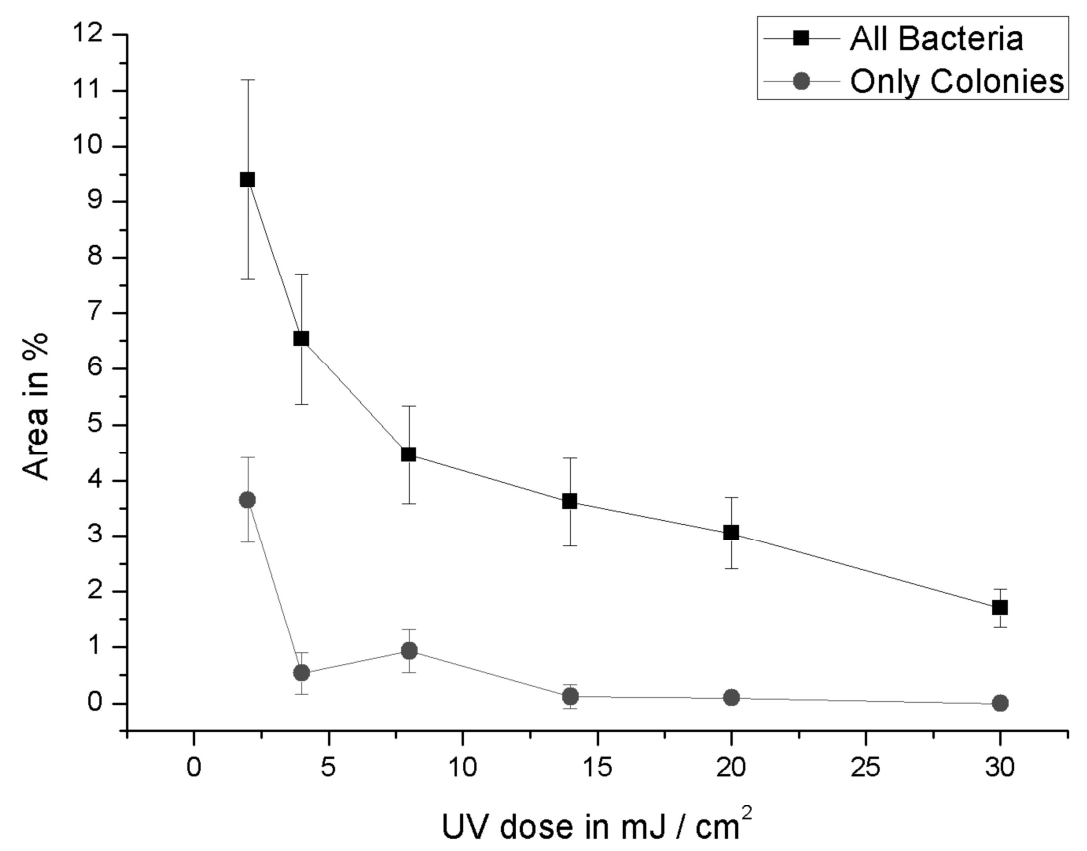

